\title{
Impact of tacrolimus intra-patient variability on kidney transplant outcomes according to immunologic risk
}

\author{
Eun Jin Kim, Juhan Lee, Soo Jin Kim, Jae Geun Lee, Beom Seok Kim, Dong Jin Joo, Kyu Ha Huh, Myoung Soo Kim,
} Soon II Kim, Yu Seun Kim

Division of Transplantation, Department of Surgery, Yonsei University College of Medicine, Seoul, Korea

Background: High intra-patient variability (IPV) of tacrolimus trough concentrations is increasingly recognized as a predictor of poor long-term outcomes in kidney transplant recipients. However, there is a lack of information regarding the impact of tacrolimus IPV on graft outcomes according to immunological risk. We hypothesized that tacrolimus IPV has different effects on graft outcomes based on the patient's individualized immunological risk.

Methods: We analyzed tacrolimus IPV using the coefficient of variability from months 6-12 after transplantation in adult kidney transplant recipients. Patients were divided into two immunological risk groups based on penal reactive antibodies and presence of donor specific antibodies.

Results: A cohort of 1,080 kidney transplant recipients with 9,059 tacrolimus trough levels were analyzed. The effects of tacrolimus IPV on graft outcomes was significantly different between low and high immunological risk patients. Overall graft survival of high immunological risk group was significantly impaired with high tacrolimus IPV $(P<0.001)$. A multivariable Cox regression confirmed high tacrolimus IPV was independent risk factor for graft loss in the high-risk group (hazard ratio [HR], 2.94; 95\% confidence interval $[\mathrm{Cl}], 1.42-6.08 ; P=0.004)$. High tacrolimus IPV was also significantly associated with increased risk of late-onset rejection in the high-risk group $(P=0.006)$. In contrast, overall graft survival and cumulative probability of late-onset rejection of low immunological risk group was not significantly different according to tacrolimus IPV. Low hematocrit at 12 months was a significant risk factor for high tacrolimus IPV.

Conclusions: Tacrolimus IPV has significant impact on late-onset rejection and graft loss in patients with high immunological risk.

Corresponding author: Juhan Lee

E-mail: laplaine@yuhs.ac

(C) The Korean Society for Transplantation

This is an Open Access article distributed under the terms of the Creative Commons Attribution Non-Commercial License (http://creativecommons.org/licenses/by-nc/4.0/) which permits unrestricted non-commercial use, distribution, and reproduction in any medium, provided the original work is properly cited. 